\title{
MS06-05 | Structural Alphabets for Conformational Analysis of Nucleic Acids
}

Cerny, Jiri (Institute of Biotechnology, Czech Academy of Sciences, Vestec, CZE); Bozikova, Paulina (Institute of Biotechnology, Czech Academy of Sciences, Vestec, CZE); Schneider, Bohdan (Institute of Biotechnology, Czech Academy of Sciences, Vestec, CZE)

We present a universal nucleic acids structural alphabet suitable for conformational analysis of DNA as well as RNA structures. The underlying local conformational classes of dinucleotide step are characterized by seven torsion angles of the sugar-phosphate backbone, two torsions around glycosidic bonds, and three parameters describing the mutual orientation of bases within the step. Our previous definition of 44 DNA classes [1,2] was extended to incorporate also RNA conformers. We have obtained 84 conformational classes covering the combined structural variability of DNA and RNA.

The newly determined set of conformers substantially extends the previously determined set of RNA conformers [3]. All the classes are defined by means of hierarchical clustering of data from a sequentially non-redundant set of high resolution crystal structures containing about 60 and 60 thousands steps for DNA and RNA, respectively.

We have found that many of the previously defined conformational classes determined as specifically DNA [1] or RNA [3] are shared among nucleic acids. The most significant feature of new conformational classes is a high number of conformers with non-stacked steps. Despite the larger diversity of the molecular architecture of RNA molecules, many unstacked ("open") conformations are also found in DNA. The assignment of nucleic acids conformation is available at https://dnatco.org as a freely accessible web service [4].

[1] Schneider et al., Acta Cryst. D 2018, 74, 52-64.

[2] Schneider et al., Genes (Basel) 2017, 8, 278.

[3] Richardson et al., RNA 2008, 14, 465-481.

[4] Černý et al., Nucleic Acids Research 2016, 44, W284. 